\title{
Development of Nano finishing technique for flat and curved plates
}

\author{
G. Parameswari*, V. K. Jain and J. Ramkumar \\ Department of Mechanical Engineering, Indian Institute of Technology Kanpur, Kanpur,UP, India \\ *Email: paramg@iitk.ac.in
}

The life and functionality of any product depend greatly on the quality of the surface produced. In the current world nano-level surface finish has become a functional requisite of many products. To finish such components without any micro/nano defects and with nano scale finishing requirements, we need a flexible finishing tool. Such finishing processes with flexible polishing tool can be classified into two categories: with and without magnetic field assistance. The former includes magnetic abrasive finishing, magnetorheological finishing, and allied processes, and the latter includes abrasive flow finishing. Ultrafine surface on three-dimensional (3D) complexshaped components and very hard material components require high specific cutting energy and loose abrasives. Thus, traditional finishing processes, such as grinding, honing, and lapping, that are abrasive based but they are not capable to finish such components due to the abrasives in the bonded form. Therefore magnetic field-assisted finishing processes are preferable over the traditional finishing processes in many cases [1].

Magnetorheological finishing (MRF) is a nano finishing process developed for obtaining super finished mirror surfaces. MRF uses a Magneto rheological polishing (MRP) fluid which consists of magnetic particles, nonmagnetic abrasive particles, and some additives in water or other carrier to polish the materials. MRP fluid acts as a Newtonian fluid in the absence of magnetic field and viscoplastic fluid in the presence of magnetic field. This exceptionally good characteristic facilitates rapid control (in milliseconds) of the yield stress, and thus the pressure applied to the workpiece surface to be polished. When the magnetically stiffened MRP fluid flows over the surface, the exposed abrasives interact with the asperities of the surface and thus results in the material removal.

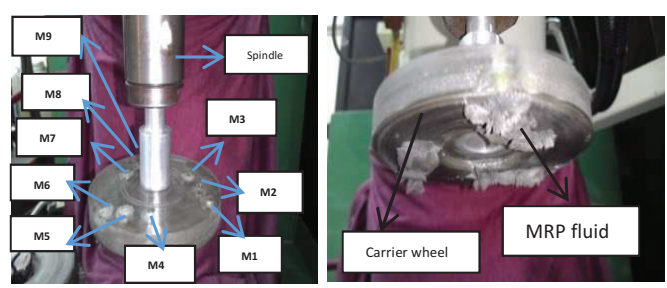

Figure 1: (a) MRF Tool (Magnetic fixture with button magnets (M1-M9)), (b) Carrier wheel with MRP fluid brush
In this study, Sintered Nd-Fe-B permanent button magnets (N48 grade) are used to provide magnetic field in the finishing zone as shown in Fig1(a). MRP fluid is applied over magnets as shown in Fig1 (b) and is rotated maintaining a constant working gap (1mm) while finishing.

A flat Titanium disc has been finished on the developed tooling. The surface roughness of the disc before $\left(\mathrm{Ra}_{\mathrm{i}}\right)$ and after finishing $\left(\mathrm{Ra}_{\mathrm{f}}\right)$ are noted at four different points on the surface with the help of a template and then averaged to get the value accurately as shown in table 1 . To normalize the effect of $\mathrm{Ra}_{\mathrm{i}}$ on the final results \% change in $\mathrm{Ra}$ is calculated (Eq 1) which will be useful in further study. The surface roughness is measured by Federal Surf-analyser 5000 Instrument and the cut-off value of $0.8 \mathrm{~mm}$ is chosen to filter waviness from the measurement. Finishing time is an interaction time of workpiece and MR fluid. Finishing rate is calculated $(\mathrm{Eq} 2)$ at three different finishing times.

$\%$ change in $\mathrm{Ra}=\frac{(\mathrm{Rai}-\mathrm{Raf})}{\mathrm{Rai}} \times 100$

Finishing rate $=\left(\mathrm{Ra}_{\mathrm{i}}-\mathrm{Ra} \mathrm{a}_{\mathrm{f}}\right) /$ finishing time

Table 1: Surface roughness before and after finishing (60 $\min$ )

\begin{tabular}{lll}
\hline \multicolumn{3}{l}{ Ra value $(\boldsymbol{\mu m})$ before and after finishing } \\
\hline POINT & $\begin{array}{l}\mathbf{R a}_{\mathrm{i}} \\
(\mu \mathrm{m})\end{array}$ & $\begin{array}{l}\text { Raf } \\
(\mu \mathrm{m})\end{array}$ \\
$\mathbf{A}$ & 0.13 & 0.0966 \\
$\mathbf{B}$ & 0.133 & 0.13 \\
$\mathbf{C}$ & 0.17 & 0.126 \\
D & 0.096 & 0.1033 \\
average & $\mathbf{0 . 1 3 2}$ & $\mathbf{0 . 1 1 4}$ \\
\hline
\end{tabular}

Table 2: \%change in $\mathrm{Ra}$ and finishing rate of the disc

\begin{tabular}{ll}
\hline \%change in Ra & $\begin{array}{c}\text { Finishing rate } \\
(\mu \mathrm{m} / \mathbf{m i n})\end{array}$ \\
\hline $\mathbf{1 3 . 6 3}$ & 0.0003 \\
\hline
\end{tabular}

Experiments are planned to carry out the study of effect of process parameters such as finishing time, working gap, volume concentration of CIPs and abrasive particles wheel rotational speed on \% change in Ra, MRR, and finishing rate. Theoretical analysis of forces acting on the surface during finishing is also being made.

\section{Reference}

1. Jain, V. K., Sidpara, A., Sankar, M.R., and Das, M. Nanofinishing techniques: a review Proc. IMechE Vol. 000 Part C: J. Mech Eng Sci 2011, DOI: 10.1177/0954406211426 